\title{
LUMINESCENCE CHARACTERIZATION OF GaAs SINGLE QUANTUM WELIS
}

R. MITDANK, H. HAEFNER, E. SCHULZE and G. OELGART*

Humboldt Universität zu Berlin, Fachbereich Physik, Institut für Ionen- und Elektronenphysik, Invalidenstr. 110, D-1040 Berlin, Germany

${ }^{*}$ Universität Leipzig, Fachbereich Physik, Linnéstr. 5, D-1070 Leipzig, Germany

\section{ABSTRACT}

The peculiarities of the photo- and cathodoluminescence of MOVPE grown single quantum wells containing impurities are investigated. The investigations reveal monolayerfluctuations with a lateral extension of about $10 \mathrm{\mu m}$. The incorporation of carbon in the thicker islands is shown using micrographs of spectrally resolved cathodoluminescence. Extrinsic radiative transitions involving the impurities are found also in the barrier above that islands which contain impurities. These inhomogenities are proposed to be connected with dislocations emanating from the substrate and penetrating the sandwiched layer structure.

\section{REFERENCES}

/1/D.Bimberg, J.Christen, T.Fukunaga, H.Nakashima, D. E. Mars, and J. N. Miller

Superlattices and Microstructures 4, 257 (1988)

/2/J.Christen

Dissertation, Technische Universität Berlin (1988)

/3/G.Bastard, Phys.Rev. B 24,4714 (1981)

/4/H.Haefner, L.Lehmann, R.Mitdank, G.Oelgart, and E.Schulze

phys. stat. sol.(a) 122,683 (1990) 


\section{INTRODUCTION}

This paper is a report of single-quantun well characterization by means of photoluminescence (PL) and cathodoluminescence (CL) investigations. These two nethods ensure complementary results with regard to the local and spectral resolution, the injection level dependencies and the imaging possibilities. About such measurements at MBE grown $S Q W ' s$ was reported in $/ 1,2 /$.

AlGaAs/GaAs/AlGaAs unintentionally doped single quantue wells (SQW) in the present work were grown by low-pressure MOVPE. The grown layer structure is shown in Fig. 1. The electronic structure of a $7 \mathrm{~nm}$ GaAs-well investigated is shown in Fig. 2 .

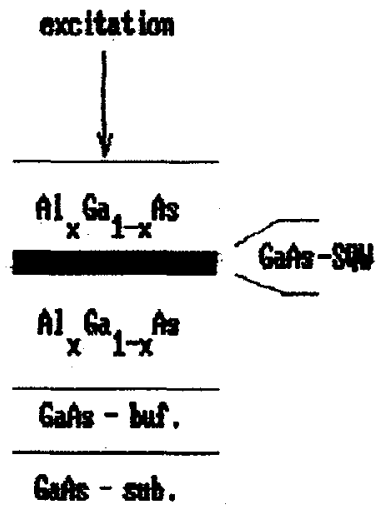

Fig. 1

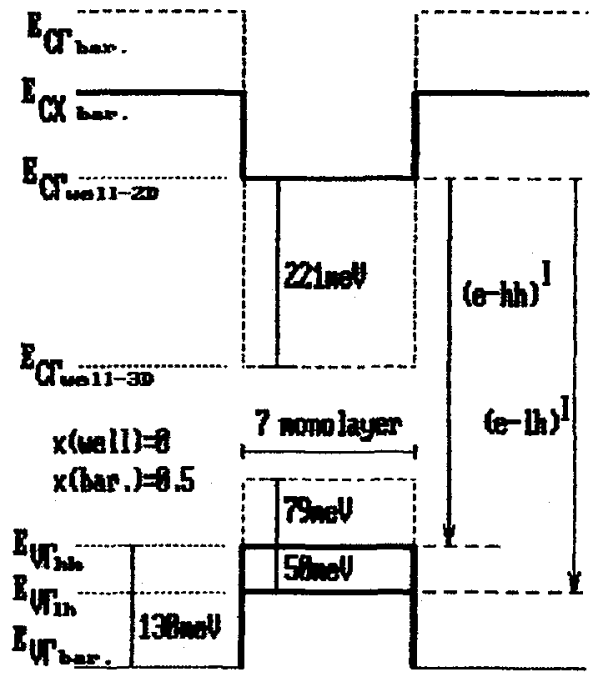

Fig. 2

CL excited with the primary electron energy of $20 \mathrm{keV}$ was measured in the temperature range between $80 \mathrm{~K}$ and $300 \mathrm{~K}$. It is interesting to note that the electron-hole pair generation function excited by the electron bean with a primary energy of $20 \mathrm{keV}$ exhibits a maximum at distances of about 0.4 um below the surfaces and decreases to about $50 \%$ at a depth of 1 um. The $632.8 \mathrm{~nm}$ line of a HeNe laser was used to carry out PL measurements. The $1.96 \mathrm{eV}$ photons penetrate the barriers without any absorption and a local resolution comparable with the laser spot diameter $(\approx 3 \mu \mathrm{m})$ can be expected.

\section{MICROPHOTOLUMINESCENCE}

In Fig. 3 typical PL-spectra taken at different positions of the GaAs-SQW are shown. 


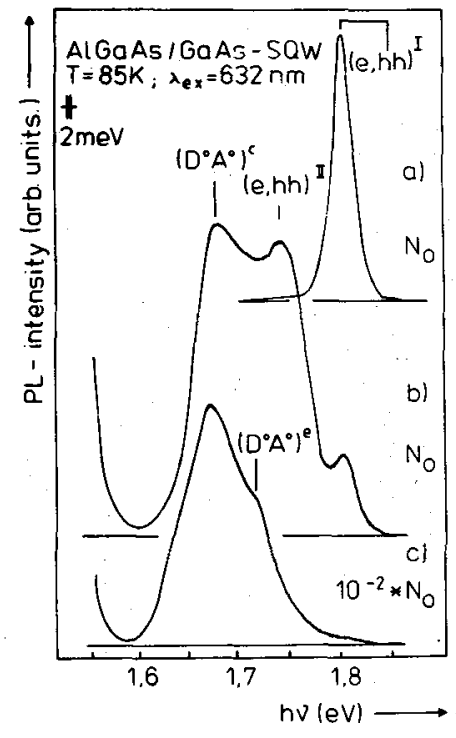

Fig - 3
Only one band due to the radiative decay of electrons and heavy holes associated with the lowest conduction and valence band aublevels $\left(h \nu_{\mathrm{c}}(e-h h)=1.806 \mathrm{eV}\right.$; FWHM = 20 meV) was observed on the regular quantum well (curve a). The luminescence band II at $1.745 \mathrm{eV}$ was obtained with the strongly focused laser beam only and appears in a number of small regions of the sample (curve $b$ and c). In those regions where band II is discernible a strong intensity of the low-energy band can be obtained also. Considering the similar behaviour of the high-energy bands I and II as the excitation density was varied and the abovementioned features of band II, we attribute the $1.745 \mathrm{eV}$ transition to a radiative decay of $2 D$ electrons and heavy holes (e-hh) between the lowest conduction and valence band sublevels of thicker SQW regions with relative small lateral extensions $(8 \mathrm{BE}=239 \mathrm{meV})$. This hypothesis will be discussed later. As the pump level is increased the low

energy band shifts to higher enersy, typical for a ( $\left.{ }^{*} A^{*}\right)$ transition in bulk

material, namely, the saturation of more distant pairs in favour of closer ones which produce higher-energy photons. For low excitation levels (curve c - $N=1 \mathrm{~W} / \mathrm{cm}^{2}$ ) donor-acceptor pair transitions connected with the centre and edge of the wellocated acceptorg were resolved $\left(h_{\nu}\left(D^{*} A^{*}\right)^{C}=1.672 \mathrm{eV}, h_{c}\left(D^{*} A^{*}\right)=1.720 \mathrm{eV}\right)$ in the impurity contaninated regions.

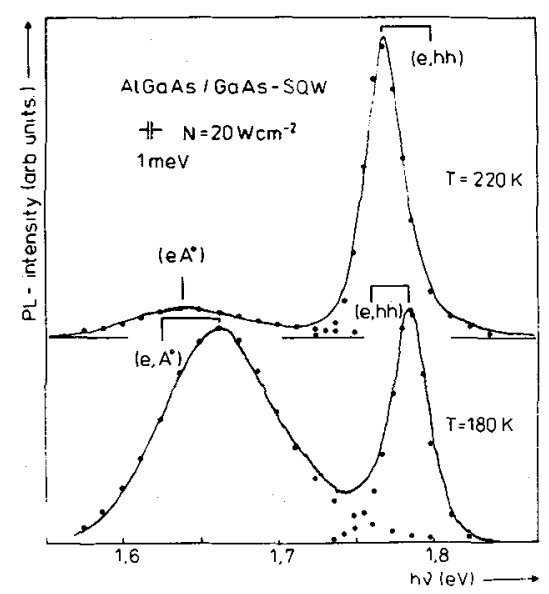

Fig . 4
At higher temperatures only the $(e-h h)$ and the low-energy extrinsic transitions were observed. Two corresponding PL spectra are displayed in Fig. 3 recorded at $T=180$ and $220 \mathrm{~K}$. The decreasing peak energy of the $(\mathrm{e}-\mathrm{hh})^{\mathrm{I}}$ bands (h) $(180 \mathrm{~K})=1.784$ $\mathrm{eV}$ and $\left.h \nu_{c}(220 \mathrm{~K})=1.767 \mathrm{eV}\right)$ corresponds to the lowering of the energy gap with increasing temperature. The impurity correlated luminescence bands reveal peak positions independent of the pump level indicating the free to bound character of the extrinsic transitions in this temperature range (not shown). We attribute this bands $\left(\mathrm{h} \nu_{\mathrm{g}}(180 \mathrm{~K})=1.659 \mathrm{eV}\right.$ and $h \cup_{c}(220 K)=1.642 \mathrm{eV}$ ) to the radiative decay of $2 D$ electrons with holes bound to acceptors located in the well centre $\left(\mathrm{eA}^{*}\right)$. 


\section{LOCAL LUMINESCENCE DISTRIBUTION}
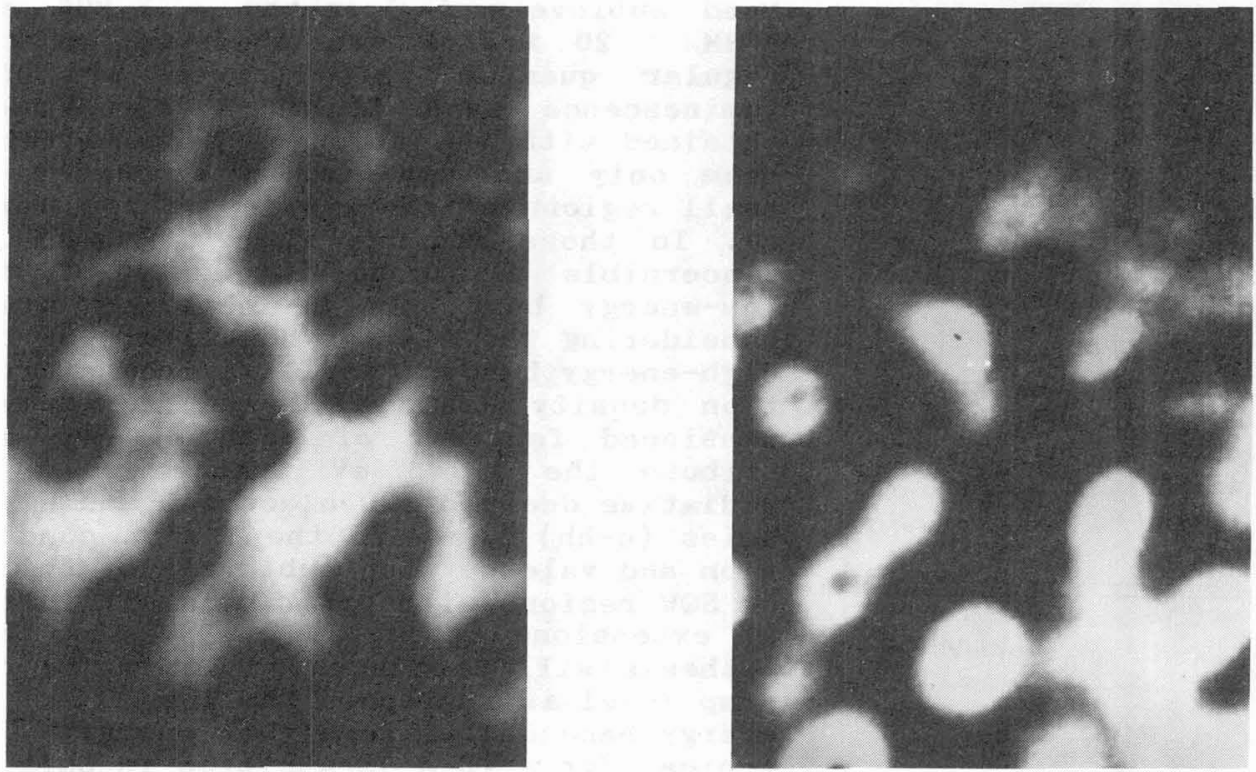

Fig. 5

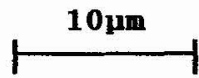

Fig. 6

Two monochromatic CL micrographs are shown in. Fig. 5 and 6 which were taken with a focused electron beam of the primary energy $E=$ $20 \mathrm{keV}$ and $T=85 \mathrm{~K}$ at the wavelengths of the $\mathrm{h}_{\mathrm{c}}(\mathrm{e}-\mathrm{hh})^{\mathrm{I}}$ (Fig. 5) and extrinsic transitions (Fig. 6 ). As a result, in the $h \nu_{c}\left(D^{\circ} A^{\circ}\right)$ picture (Fig. 6) the white islands contrast the local ippurity distribution. The visible islands exhibit an extension up to $10 \mu \mathrm{n}$. An overlapping of the different contrast regions results from the carrier diffusion in the barrier and in the well. The overlapping is about $1.5 \mathrm{~km}$. The striking feature is the complementary behaviour of the pictures taken with the peak wavelength of the $h p_{c}(e-h h)$ band and extrinsic transitions indicating the incorporation of the inpurities in single regions of the quantum well with diameterg in the lim range.

Additionally we measured the luminescence spectra excited with the electron beam in impurity contaminated regions. Two corresponding CL spectra recorded at $T=85 \mathrm{~K}$ are displayed in Fig. 7 . The energy scale is listed with respect to the 3D energy gap of GaAs. The broad luminescence band generated on the impurity contaminated ialands (dashed line) is caused by the convolution of the (e-hh) II and $\left(D^{\circ} A^{\circ}\right)^{C}$ bands. The CL spectra outside of these islands (solid line) are governed by $a$ band due to the $(e-h h)^{I}$ transition. The low energy shoulder indicates the diffusion of excess carriers to the impurity contaminated regions. 


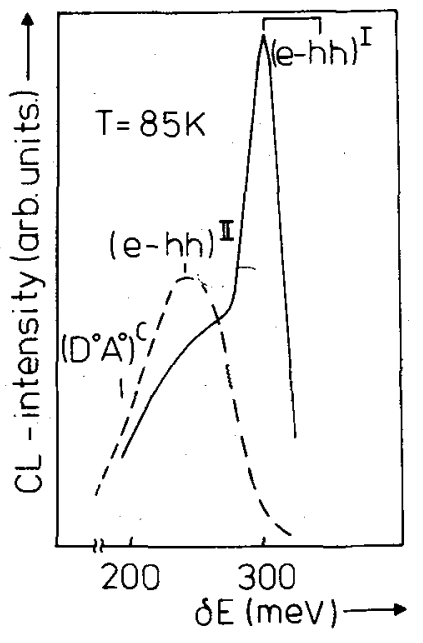

Fig. 7

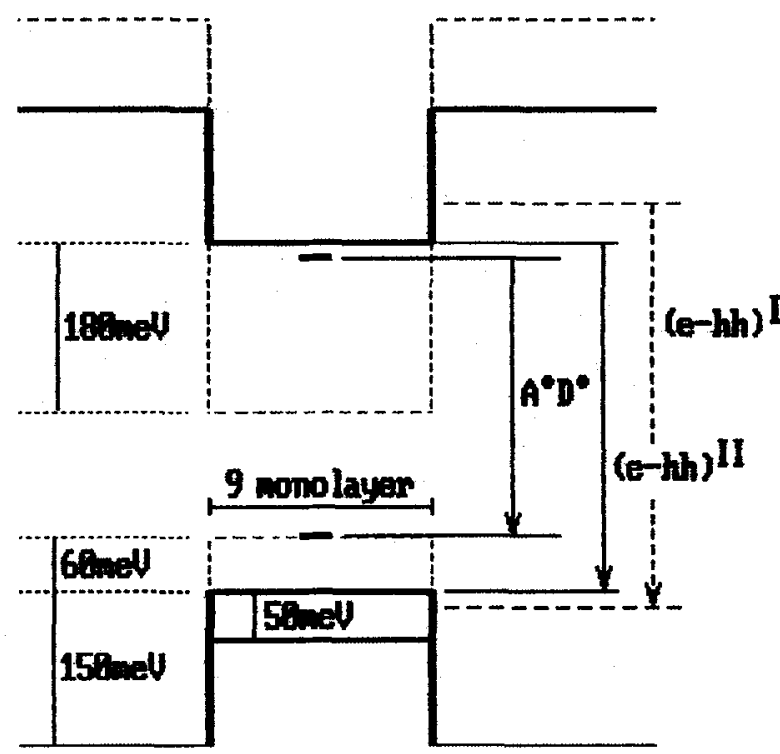

Fig - 8

As a result, residual impurities were found in small quantur well regions, which realize a weaker confinement of the carriers than found in the regular quantum well. The observed transitions are shown in Fig. 8 ( straight line:regular SQW, fulf line:impurity contaminated region). From the peak shift $8 \mathrm{E}(\mathrm{e}-\mathrm{hh}) \mathrm{f} * 300$ weV we conclude a seven monolayer thick regular quantum well. The lower confinement in the small quantum well regions could be due to a nine monolayer thick well.

\section{THE NATURE OF THE IMPURITY}

Although the chemical nature of the acceptor found in the saw has not yet been determined, it is thought to arise from carbon on As site, since it usually appears in material prepared by MOCVD in GaAs and AlGaAs alloys. Taking the lowest energies at which the extrinsic peaks were observed in excitation dependent PL investigations at $T=$ $85 \mathrm{~K}\left(\mathrm{~h} \nu_{\mathrm{C}}\left(\mathrm{D}^{\circ} \mathrm{A}^{*}\right)^{\mathrm{C}}=1.672 \mathrm{eV}\right)$ as the peak position in the low excitation limit the total activation energy of the acceptors and donors at the well centre was found as $74 \mathrm{meV}$. From the free to bound transition at temperatures $T>110 \mathrm{~K}$ the ionisation energy value of the well centre acceptor can be determined using the relation

$$
\mathbf{E}_{\mathbf{a}}^{\mathbf{c}}=\mathbf{E}_{\mathbf{s b}}-\mathbf{h} \nu_{\mathbf{c}}(\mathbf{e A})+\frac{1}{2} \mathbf{k T}
$$

where $\mathrm{E}_{\mathrm{sb}}$ denotes the energy distance between the subband levels and $\frac{1}{2} k T$ results from the thermal distribution of the free carriers in the subband. The extrinsic bands appear in connection with the $\left(e^{-h h}\right)^{I I}$ transition. Consequently, we estimated the acceptor ionisation energy with regard to the heavy hole sublevel energy in the nine-monolayer sQW. Using (1) the ionisation energy of the acceptor located in the centre of the well amounts to $63 \mathrm{meV}$. 


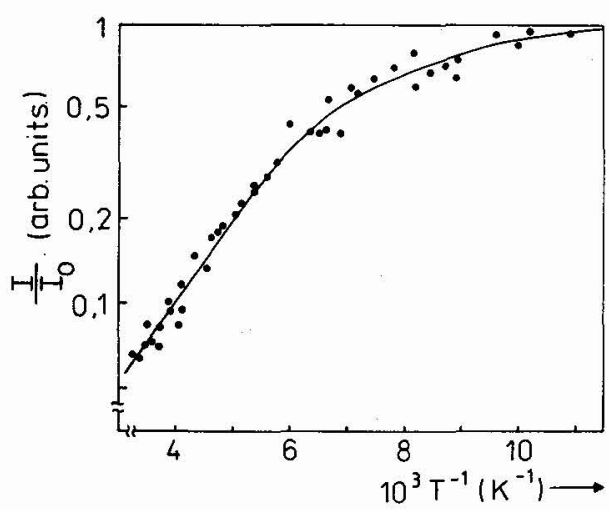

Fig. 9
This assignment was confirmed by the temperature dependence of the (eA ) transition CL intensity. The intensity versus the inverse teeperature is listed in Fig. 9. The theoretical curve was calculated using the relation for a two-level model:

$I\left(e A^{*} ; T\right)=I_{0}\left[1+A \exp \left(-E_{a}^{c} / k t\right)\right]^{-1}$

The activation energy evaluated from Fig. 9 anounts to $\mathrm{E}_{\mathrm{a}}=$ $63 \mathrm{meV}$ in excellent agreement with the above found result. From the measurements a donor activation energy of $11 \mathrm{eeV}$ in reasonable agreement with the expectation for a donor localized in the well

$/ 3 /$ can be concluded.

\section{DISCUSSION}

Fig. 10 and Fig. 11 illustrate the local distribution of the extrinsic luminescence in the barrier and in the $S Q W$ at $T=90 \mathrm{~K}$. It is interesting to note that the luninescence due to extrinsic transitions in the barrier and in the quantum well appears at the same lateral positions. Around these inpurity containing regions the (e-hh) ${ }^{I}$ transition dominates in the sQW and the free exciton luminescence band correlates with the $x$-niniwum of the conduction band in the barrier /4/.

As a result, we conclude a wide regular seven nonolayer thick quantum well with excellent interface quality. This well involves ninemonolayer thick impurity contaninated regions with lateral extensions in the micrometer range. Locally correlated impurity contaninated regions were also observed in the barriers and we conclude dislocations which grow through the wafer enanating from the substrate. The dislocation density in the range between $10^{3}$ and $10^{4} \mathrm{~cm}^{-2}$ corresponding to values found on low-quality substrates confirms this hypothesis.
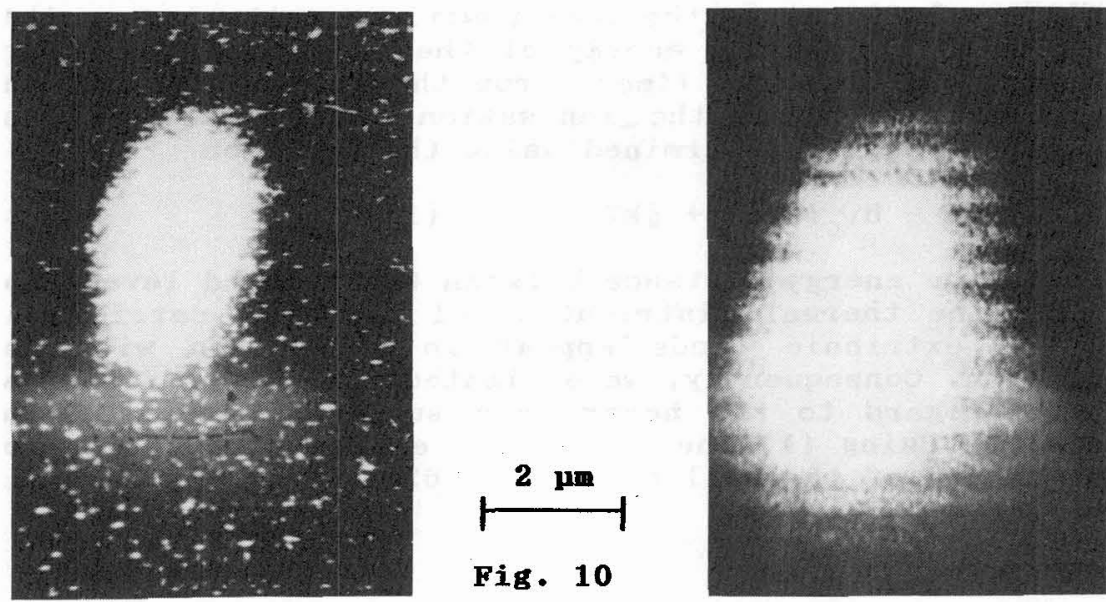

Fis. 11 